\title{
Olfactory neuroblastoma - a rare tumour under the guise of chronic rhinosinusitis
}

\author{
Grażyna Stryjewska-Makuch, Marcelina Niemiec-Urbańczyk, Bogdan Kolebacz
}

Department of Laryngology and Laryngological Oncology, Upper Silesian Medical Centre, Independent Public Research Hospital No. 7 of Silesian Medical University, Katowice, Poland

Olfactory neuroblastoma (ONB), also called esthesioneuroblastoma, is a rare tumour that occurs in the nasal cavity and paranasal sinuses. The symptoms that patients report are similar to chronic rhinosinusitis (CRS). The authors present the case of a patient referred to hospital due to nasal obstruction on the left side. The performed endoscopic surgery revealed within the left middle nasal concha a tumour penetrating into the anterior ethmoid and frontal recess. ONB was diagnosed in the histopathological examination. The patient was qualified for complementary radiotherapy. The patient has been under medical supervision for 24 months. There have been no signs of recurrence. It was decided to present the above case due to the lack of deviations in the ENT endoscopy and minor ailments reported by the patient, which in combination with advanced age could lead to discontinuation of surgical treatment.

NOWOTWORY J Oncol 2019; 69, 5-6: 184-186

Key words: esthesioneuroblastoma, endoscopic sinus surgery (ESS), skull base, sinonasal

\section{Introduction}

Olfactory neuroblastoma (ONB), also called esthesioneuroblastoma, is a rare tumour, occurring in 0.4 to 1 case per million [1], and accounts for 3-6\% of all malignant tumours of the nasal cavity and sinuses [2]. The tumour is locally malignant, infiltrates the nasal cavity, sinuses, cribriform plate, orbit and cranial cavity.

The origin of the tumour is controversial. It is believed that the starting point of tumour cells are primary olfactory epithelial progenitor cells. The neuronal origin of the tumour or the origin from the neural crest cells is demonstrated primarily by the presence of neurofilaments [3].

In order to determine the clinical advancement of ONB, the Kadish staging system from A to D [4] is most commonly used. In situ tumour, restricted to the nasal septum, floor, lateral wall, nasal passage, nasal concha or nasal vestibule with/without bone infiltration is designated as stage A. A tumour stretching beyond paranasal sinuses or extending to the sinuses or an ostiomeatal complex is classified as stage B. Infiltration of the skull base, palate, cribriform plate, medial wall or orbital wall, pterygoid plates, nasopharynx, skin, dura mater and brain is qualified as grade C. Patients with the involvement of lymph nodes or internal organs are classified as grade D.

The TNM modification proposed by Dulguerov and Calcaterrain [5] is also used:T1 - tumour involves the nasal cavity and/or sinuses (except the sphenoid sinus), ethmoid cells, T2 - tumour involves the nasal cavity and/or sinuses along with the sphenoid sinus, with infiltration or erosion of the cribriform plate, T3 - tumour infiltrates the orbital tissue or spreads to the front of the skull base, T4 - tumour spreads to the brain, No - no cervical lymph node metastases, N1 - any form of cervical lymph node metastases, M0 - no metastases, M1 distant metastases. A similar classification is proposed by the American Joint Committee on Cancer (AJCC) that separately describes the tumour invasion in the TNM scale depending on the tumour origin, within the nasal cavity and ethmoid cells, or in the maxillary sinus [6]. Unfortunately, Kadish staging systems, Dulguerov's modified TNM, and AJCC TNM, all poorly depict 
patient prognosis over a 10-year horizon [6]. Histopathological evaluation of the tumour is based on the Hyam's classification in stages from I to IV [7].

Clinical symptoms are varied. The most common ones include nasal obstruction (90\%), preceded by bleeding (30\%). Anosmia, headaches, facial pain, sinusitis, diplopia, epiphora, proptosis with the orbit involvement are less frequent [8,9].

Kuan [10], in a retrospective analysis of data from 40 years, found that in 841 patients with ONB the tumour originated from the nasal cavity in $83 \%$ of cases, followed by the ethmoid sinus $-3.9 \%$, maxillary sinus $-1.3 \%$, sphenoidal sinus $-0.7 \%$ and frontal sinus $-0.35 \%$. The presence of lymph node metastases was quite rare (less than 10\%), most commonly these were group II lymph nodes. There was no relationship between the presence of lymph nodes metastases and the primary location of the tumour. No recommendation can be made about the elective neck dissection or radiation therapy of the NO neck [11].

High-resolution magnetic resonance imaging, thin-cut maxillofacial computed tomography (CT), and fluorodeoxyglucose positron emission tomography combined with $\mathrm{CT}$ were performed preoperatively in each case.

\section{Case report}

In August 2017, a 74-year-old patient was admitted to the Department of Laryngology and Laryngological Oncology due to nasal obstruction on the left side. The patient did not report any pain, nasal bleeding or recurrent inflammation. Tomographic examination of the sinuses revealed abnormal soft-tissue masses with the presence of calcification in the left middle nasal concha penetrating into the anterior ethmoid, frontal recess and nasolacrimal canal. The left frontal sinus was airless. The size of the lesion was $29 \times 20 \times 24$ mm (Fig. 1 and 2). The patient suffered from hypertension, varicose veins

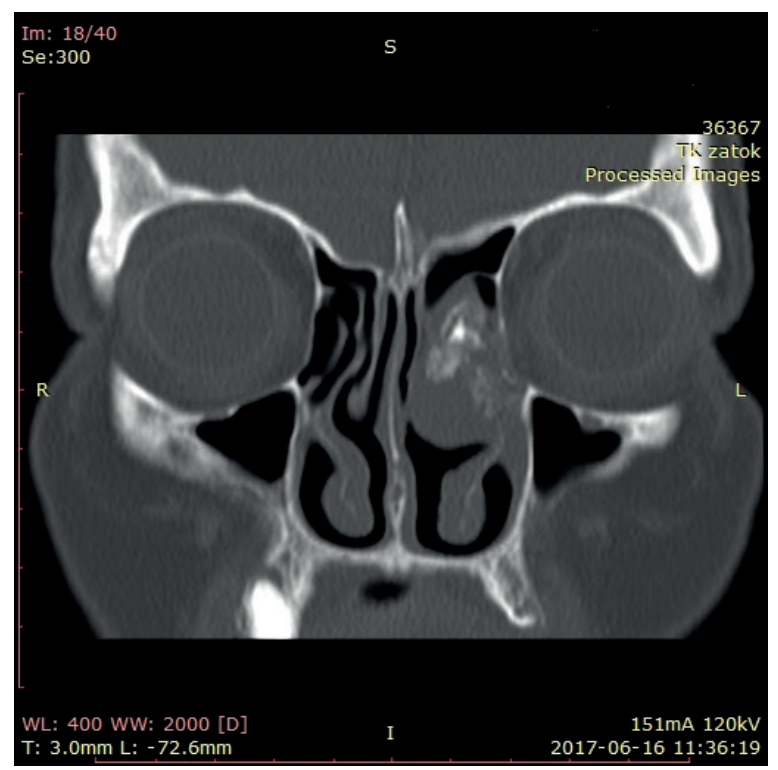

Figure 1. CT scan before the surgery

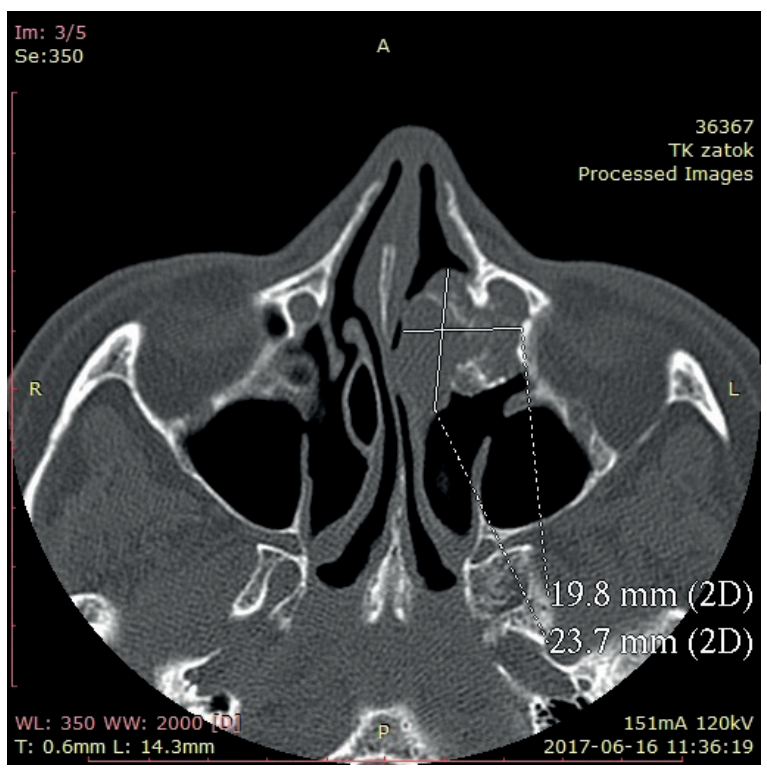

Figure 2. The size of the lesion in CT scan

and ulceration of the lower limbs. Endoscopic examination revealed a distended, enlarged middle nasal concha. Based on the tomographic examination, the extent of the lesion was estimated at 6 points on the Lund-Mackay scale.

No enlarged lymph nodes were found in the palpation and radiological examination. Endoscopic sinus surgery (ESS) was performed under general anesthesia with hypotension. The distended, enlarged left middle nasal concha was incised and granular tissue masses suggesting inverted papilloma were collected. The lateral lamella of the concha, uncinate process were removed, the maxillary sinus ostium was widened. The sinus cavity was clean. Then the anterior ethmoid and frontal recess were opened. The left frontal sinus was filled with mucus content. There were no deviations from the normal state on the right side of the nasal cavity. Material for histopathological and bacteriological examination was collected.

Histopathological examination revealed olfactory esthesioneuroblastoma, Hyam's grade II EMA(-), CK(-), VIM(-), LCA(-), ABpaS(-), Ki <5\%, NSE(+), SNPF(+), S100(-), ChromA(+). The lesion in the Kadish scale was grade $B$ (despite originating from the middle concha, it spread to the frontal recess), in the TNM scale - T1NOMO scale. Escherischia coli, Staphylococcus hominis were cultured. After the oncological consultation, a decision was taken to extend the procedure. ESS was performed, removing the remainder of the middle concha, opening widely the ethmoid and frontal recess, removing the orbital lobe and clearing the ethmoid roof. Margins were collected stating tumour infiltration in the rest of the middle concha and frontal recess. The patient was qualified for complementary radiotherapy.

The patient is examined endoscopically every 3 months and radiologically every 6 months. Up to now, 24 months after surgery, there have been no recurrent tumour features (Fig. 3). 


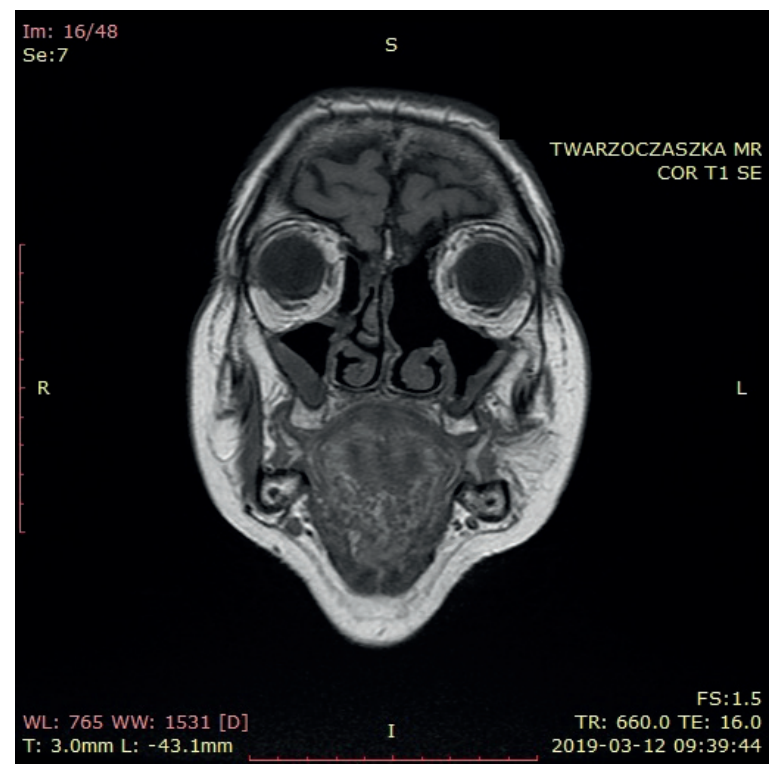

Figure 3. MR scan 24 months after surgery

\section{Discussion}

The generally accepted method of ONB treatment is surgery with subsequent radiotherapy. Surgical techniques include transnasal craniofacial resection with/without craniotomy or expanded endoscopic endonasal craniofacial resection, depending on the tumour location and extension [5, 11]. The development of endoscopic techniques allows for safe resection of advanced tumours, and preservation of olfactory function may be considered for patients with ONBs showing no invasion in the contralateral side $[9,12]$. Adjuvant chemotherapy is sometimes added for high-grade, recurrent, or locoregionally advanced tumours [11, 13]. The comparison of surgical treatment, chemotherapy and radiotherapy, as the only therapy combined with surgical methods, showed that better results were obtained in the case of surgery and subsequent radiotherapy $[1,14]$.

It has been found that a bad prognostic factor is the high grade in Hyam's scale and the presence of lymph nodes metastases. Examining the results of the 5-year and 10-year survival, it was observed that patients in stage A lived shorter than in stage B, regardless of whether they were treated only surgically or in combination with subsequent radiotherapy.

Perhaps this is the result of a more radical surgical treatment of B-stage tumours [2]. A retrospective analysis of 876 patients showed that an important prognostic factor for ONB recurrence was age $>60$ years [15]. Patients require regular oncological and laryngological follow-ups. Magnetic resonance imaging after surgery is suggested every 3 months in the first year after surgery, regardless of the regular endoscopic examination of the nasal cavity.
The described case suggests the importance of performing accurate endoscopic examination of the nasal cavity in combination with computed tomography in patients over 60 , reporting even minor disturbances, especially one-sided. Early surgical intervention gives the patients a chance to be treated definitively.

\section{Conflict of interest: none declared}

\section{Marcelina Niemiec-Urbańczyk}

Independent Public Research Hospital No. 7 of Silesian Medical University

Upper Silesian Medical Centre

Department of Laryngology and Laryngological Oncology

ul. Ziołowa 45

40-635 Katowice, Poland

e-mail:marcelina.niemiec@gmail.com

\section{Received: 27 Sep 2019}

Accepted: 25 Nov 2019

\section{References}

1. Gram SB, Gronhoj Ch, Mann H et al. Patient characteristics of olfactory neuroblastoma: experience from a tertiary cancer centre 2000-2016 covering Estern Denmark. APMIS. 2018; 126 (8): 663-666.

2. Konuthula N, Lloreta AM, Miles B et al. Prognostic significance of Kadish staging in esthesioneuroblastoma: an analysis of the National Cancer Database. Head Neck. 2017; 39 (10): 1962-1968.

3. Dulguerov P, Allal AS, Calcaterra TC. Esthesioneuroblastoma: a meta-analysis and review. Lancet Oncol. 2001; 2 (11): 683-690.

4. Kadish S, Goodman M, Wang CC. Olfactory neuroblastoma. A clinical analysis of 17 cases. Cancer. 1976; 37 (3): 1571-1576.

5. Dulguerov $\mathrm{P}, \mathrm{Cal}$ caterra T. Estesioneuroblastoma: the UCLA experience 1970-1990. Laryngoscope. 1992; 102 (8): 843-849.

6. Joshi RR, Husain Q, Roman BR et al. Comparing Kadish, TNM, and the modified Dulguerov staging systems for esthesioneuroblastoma.J J Surg Oncol. 2019; 119 (1): 130-142.

7. Hyams VJ. Tumors of the upper respiratory tract and ear, in: VJ Hyams, Batsanis JG Micheals (Eds), Atlas of Tumor Pathology, 2nd ed., Armed Force Institute of Pathology, Washington DC. 1988; 240-248.

8. Lund VJ, Howard DJ, Wei WI et al. Olfactory neuroblastoma: past, present, and future. Laryngoscope. 2003; 113 (3): 502-507.

9. Manthuruthil C, Lewis J, McLean C et al. Endoscopic endonasal management of olfactory neuroblastoma: a retrospective analysis of 10 patients with quality-of-life measures. World Neurosurgery. 2016; 90: 1-5.

10. Kuan EC, Nasser HB, Carey RM et al. A population-based analysis of nodal metastases in esthesioneuroblastomas of the sinonasal tract. Laryngoscope. 2019; 129 (5): 1025-1029.

11. Nabili V, Kelly DF, Fatemi $\mathrm{N}$ et al. Transnasal, transfacial, anterior skull base resection of olfactory neuroblastoma. Am J Otolaryngol. 2011; 32 (4): 279-285.

12. Gallia GL, Asemota AO, Blitz AM et al. Endonasal endoscopic resection of olfactory neuroblastoma: an 11-year experience. J Neurosurg. 2018; 1:1-7.

13. Tajudeen BA, Ashi A, Suh JD et al. Esthesioneuroblastoma: an update on the UCLA experience, 2002-2013. J Neurol Surg B Skull Base. 2015; 76 (1): $43-49$.

14. Xiong $\mathrm{L}$, Zeng $\mathrm{X}$, Guo Ch-K et al. Optimal treatment and prognostic factors for esthesioneuroblastoma: retrospective analysis of 187 Chinese patients. BMC Cancer. 2017; 17: 254.

15. Yin $Z$, Wang $Y$, Wu Y et al. Age distribution and age-related outcomes of olfactory neuroblastoma: a population-based analysis. Cancer Manag Res. 2018; 29: 10: 1359-1364. 